\title{
Relationship between starch and lipid accumulation induced by nutrient depletion and replenishment in the microalga Parachlorella kessleri
}

Bruno Fernandes ${ }^{\mathrm{a}}$, José Teixeira ${ }^{\mathrm{a}}$, Giuliano Dragone ${ }^{\mathrm{a}}$, António A. Vicente ${ }^{\mathrm{a}}$, Shigeyuki

Kawano $^{\mathrm{b}}$, Kateřina Bišovác, Pavel Přibyl ${ }^{\mathrm{d}}$, Vilém Zachleder, ${ }^{\mathrm{c},}$, Milada Vítovác

${ }^{\mathrm{a}}$ Institute for Biotechnology and Bioengineering, Centre of Biological Engineering, University of Minho, Campus de Gualtar, 4710-057 Braga, Portugal

${ }^{b}$ University of Tokyo, Department of Integrated Biosciences, Graduate School of Frontier

Sciences, 5-1-5 Kashiwanoha, Kashiwa, Chiba 277-8562, Japan

'Institute of Microbiology, AS CR, Laboratory of Cell Cycles of Algae, Opatovický mlýn, 37981 Třeboň, Czech Republic

${ }^{\mathrm{d}}$ Institute of Botany, AS CR, Algological Centre and Biorefinery Research Centre of Competence, Dukelská 135, 37982 Třeboň, Czech Republic

ARTICLE INFO 
Keywords:

Lipids,

Nutrient depletion,

Nutrient replenishment,

Parachlorella kessleri,

Starch

E-mail address: zachleder@gmail.com (V. Zachleder) 


\section{ABSTRACT}

Photosynthetic carbon partitioning into starch and neutral lipids, as well as the influence of nutrient depletion and replenishment on growth, pigments and storage compounds, were studied in the microalga, Parachlorella kessleri. Starch was utilized as a primary carbon and energy storage compound, but nutrient depletion drove the microalgae to channel fixed carbon into lipids as secondary storage compounds. Nutrient depletion inhibited both cellular division and growth and caused degradation of chlorophyll. Starch content decreased from an initial value of 25 , to around $10 \%$ of dry weight (DW), while storage lipids increased from almost zero to about $29 \%$ of DW. After transfer of cells into replenished mineral medium, growth, reproductive processes and chlorophyll content recovered within 2 days, while the content of both starch and lipids decreased markedly to 3 or less $\%$ of DW; this suggested that they were being used as a source of energy and carbon.

\section{Introduction}

During photosynthetic processes, over short periods of time (about 5 days), some microalgae accumulate significant quantities of lipids (60\% of DW) (Li et al., 2013) and carbohydrates (55 \% of DW) (Brányiková et al., 2011; Yao et al., 2013). These can be commercially processed into biofuels, particularly biodiesel (Yang et al., 2011) and bioethanol (Fernandes et al., 2012). However, microalgal lipids are valuable not only from the viewpoint of renewable energy, but they can also be used for the production of biochemicals, nutraceuticals, cosmetics or food-additives. Several studies have demonstrated that it is possible to control cell metabolism to yield a high content of energy-rich compounds; either starch (Brányiková et al., 2011; Dragone et al., 2011) and/or lipids (Chen 
et al., 2011; Lee, 2011). Although the mechanism of induction of lipid accumulation can be different from that of starch, there are several common approaches to induce both starch and lipid overproduction (Brányiková et al., 2011; Li et al., 2013). Lipid content can be increased by nitrogen or phosphate limitation (Hsieh and Wu, 2009; Rodolfi et al., 2009), high salt concentrations (Takagi et al., 2006), high iron concentrations (Liu et al., 2008) or growth under heterotrophic/mixotrophic culture conditions (Heredia-Arroyo et al., 2010; Shen et al., 2010).

Accumulation of starch can be induced by nitrogen depletion (Dragone et al., 2011), sulfur depletion, high light intensity (Brányiková et al., 2011) or a high $\mathrm{CO}_{2}$ concentration (Izumo et al., 2007). It was also shown that algal strains appropriate for overproducing starch are not usually suitable for overproducing lipids and vice versa (Li et al., 2013; Li et al., 2010). The microalga Parachlorella kessleri, strain CCALA 255, is characterized by a high growth rate, tolerance to high temperatures, resistance to shear stress, poor adhesion to bioreactor surfaces and a low tendency to form aggregates; this was previously tested in a large-scale thin-layer bioreactor to simulate the industrial production of microalgal lipid-rich biomass (Li et al., 2013). These are positive characteristics for its use in large-scale production bioreactors, with a potential for biofuel production. Under optimal conditions, the strain is characterized by energy storage in the form of starch rather than lipid (Li et al., 2013). If untreated, the cultures propagate rapidly, producing large amounts of biomass in a relatively short period of time. The cells contained negligible lipid storage ( $1-10 \%$ of DW) but it was possible to induce hyper-production of storage lipids in P. kessleri biomass using various methods (Li et al., 2013; Přibyl et al., 2012).

Under favorable growth conditions, algae synthesize fatty acids principally for esterification into glycerol-based polar lipids, the major constituents of intracellular membranes. However, under unfavorable environmental or stress conditions, many algae 
alter their lipid biosynthetic pathways towards the formation and accumulation of neutral lipids, mainly in the form of triacylglycerol (TAG) (Breuer et al., 2012; Li et al., 2011). These storage neutral lipids (especially TAGs) are the preferred lipids for most applications, since they can be overproduced up to very high cellular levels. TAGs have potential especially for biodiesel production, since they can be readily converted to biodiesel through existing oil refining processes (Hu et al., 2008).

It is known that alterations in nutrients can modify both growth and secondary metabolism of microalgae (Behrens and Kyle, 1996; Hsieh and Wu, 2009). Furthermore, microalgal growth depends not only on an adequate supply of essential macronutrients (carbon, nitrogen, phosphorus) and major ions $\left(\mathrm{Mg}^{2+}, \mathrm{Ca}^{2+}, \mathrm{Cl}^{-}\right.$, and $\left.\mathrm{SO}_{4}{ }^{2-}\right)$ but also on a number of micronutrient metals such as iron, manganese, zinc, cobalt, copper, and molybdenum (Dragone et al., 2011; Sunda et al., 2005). Since reduction in nutrient supply is a simple and inexpensive methodology, medium dilution (5 and 10 times) was used to increase the lipid content in P. kessleri.

Regulatory mechanisms that control the accumulation of starch and lipid in response to changes in growth conditions, and possible interactions between storage and consumption of starch and lipid are unclear (Li et al., 2010; Rawsthorne, 2002; Siaut et al., 2011). According to Siaut et al. (2011) improving microalgal strain performance requires a sound understanding of the mechanisms and regulation of carbon fixation, carbon allocation between biosynthetic pathways and induction under adverse growth conditions. Therefore, the aim of this work was to describe photosynthetic carbon partitioning between starch and neutral lipid in P. kessleri, i.e. the temporal relationship between accumulation/consumption of starch and lipid in response to nutrient depletion and subsequent replenishment. Variations in starch and lipid concentrations are compared with the concentrations of pigments and values of other cellular growth parameters, in order to elucidate how photosynthetic carbon 
partitioning between starch and lipid is affected by growth conditions that are known to induce neutral lipid production.

\section{Methods}

\subsection{Strains and growth conditions}

The green microalga Parachlorella kessleri (Krienitz et al., 2004), strain CCALA 255, was provided by the Culture Collection of Autotrophic Organisms (CCALA) in Třebon̆, Czech Republic (http://ccala.butbn.cas.cz/index.php). In the collection, the strain was

maintained on agar slants under an irradiance of about $23 \mu \mathrm{mol} \mathrm{m} \mathrm{m}^{-2}, 12 / 12 \mathrm{~h}$ (light/dark) regime and at a temperature of $12-15^{\circ} \mathrm{C}$.

Experimental cultures were prepared by transfer of algal inoculum from an agar slant into liquid mineral medium and pre-cultured in laboratory photobioreactors (see next chapter) at $30{ }^{\circ} \mathrm{C}$ in continuous light $\left(1200 \mu \mathrm{mol} \mathrm{m} \mathrm{s}^{-2} \mathrm{~s}^{-1}\right)$, in order to obtain a sufficient concentration of cells for subsequent experiments. A quantum/radiometer/photometer (LI-COR, Inc., U.S.A.) was used to measure light intensity.

The resulting cultures ("starting inocula") were transferred into fresh complete mineral medium (medium 1$)$ or 5-fold ( 0.2 medium) or 10 -fold diluted medium ( 0.1 medium) in order to obtain an initial cell concentration of $0.2 \mathrm{~g} \mathrm{~mL}^{-1}$. The control growth medium (medium 1), based on the chemical composition of the microalgal biomass (Douskova et al., 2009) had the following composition (mM): $18.32\left(\mathrm{NH}_{2}\right)_{2} \mathrm{CO}, 1.74 \mathrm{KH}_{2} \mathrm{PO}_{4}, 0.83$

$\mathrm{MgSO}_{4} .7 \mathrm{H}_{2} \mathrm{O}, 0.79 \mathrm{CaCl}_{2}, 0.11 \mathrm{FeNa}-\mathrm{C}_{10} \mathrm{H}_{12} \mathrm{O}_{8} \mathrm{~N}_{2}, 0.017 \mathrm{MnCl}_{2} .4 \mathrm{H}_{2} \mathrm{O}, 0.013 \mathrm{H}_{3} \mathrm{BO}_{3}, 0.009$ $\mathrm{ZnSO}_{4} .7 \mathrm{H}_{2} \mathrm{O}, 0.004 \mathrm{CuSO}_{4} .5 \mathrm{H}_{2} \mathrm{O}, 0.002 \mathrm{CoSO}_{4} .7 \mathrm{H}_{2} \mathrm{O}, 0.0001\left(\mathrm{NH}_{4}\right)_{6} \mathrm{Mo}_{7} \mathrm{O}_{24} .4 \mathrm{H}_{2} \mathrm{O}$ and $0.0001\left(\mathrm{NH}_{4}\right) \mathrm{VO}_{3}$ in distilled water. All culture experiments were performed at $30^{\circ} \mathrm{C}$. 
During two independent experiments, the following parameters were monitored: chlorophyll $(\mathrm{a}+\mathrm{b})\left(\mathrm{pg}\right.$ cell $^{-1}$ and $\left.\mathrm{mg} \mathrm{L}^{-1}\right)$, cell number (per $\left.\mathrm{mL}\right)$, mean cell volume $\left(\mu \mathrm{m}^{3}\right)$, dry

weight $\left(\mathrm{g} \mathrm{L}^{-1}\right)$, relative starch content (\% of dry weight (DW)), total relative lipid content (all intracellular lipids) (\% of DW) and storage (or neutral) lipid content (\% of DW).

\subsection{Laboratory photobioreactor}

The glass culture vessels were flat and rectangular in shape (inner dimensions 40 x $30 \mathrm{x}$ $2 \mathrm{~cm}$ ) with a capacity for $2000 \mathrm{ml}$ of algal suspension. Cultures were aerated and mixed vigorously with $2 \% \mathrm{CO}_{2}(\mathrm{v} / \mathrm{v})$-enriched air using a pressure can. The air mixture was delivered into the suspension by 30 bubble streams originating from a perforated stainless steel tube located at the bottom of the vessel. The gas mixture flow rate was $1000 \mathrm{~mL} \mathrm{~min}^{-1}$. This flat-vessel design enabled uniform illumination over the whole volume of a well-mixed and aerated microalgal culture. Continuous irradiance of $1200 \mu \mathrm{mol} \mathrm{m}^{-2} \mathrm{~s}^{-1}$ on the photobioreactor surface was provided by a panel of light tubes Osram L 36W/830 Lumilux (Osram, Germany); a temperature of $30 \pm 0.5^{\circ} \mathrm{C}$ was maintained using a thermostatic water bath (Fig. 1). Distilled water was added daily to the cultures to replenish that lost by evaporation.

\subsection{Biomass determination}

For dry weight determination, biomass was separated from the medium by centrifugation of $2 \mathrm{~mL}$ of the cell suspension in pre-weighed microtubes at $3000 \mathrm{~g}$ for $5 \mathrm{~min}$; the sediment was dried at $105^{\circ} \mathrm{C}$ for $12 \mathrm{~h}$ and weighed on an analytical balance Sartorius TE214S-0CE (Sartorius, Göttingen, Germany). Cell volume and concentration were measured using a 
Beckman Coulter Multisizer III (Coulter Corporation, Miami, USA) by diluting $10-50 \mu \mathrm{L}$ of fixed $(0.25 \%$ glutaraldehyde) cell suspension into $10 \mathrm{~mL}$ of $0.9 \% \mathrm{NaCl}(\mathrm{w} / \mathrm{v})$ electrolyte solution.

\subsection{Transfer to control medium}

To follow the recovery of nutrient-starved cells (after nutrient depletion on the $9^{\text {th }}$ and

$7.5^{\text {th }}$ day of culture, respectively), the original mineral medium (initially with 20 or $10 \%$ of the control nutrient concentration) was removed from the cell suspension by centrifugation and the cells were resuspended in control complete mineral medium (medium 1) to supply a concentration of nutrients high enough for recovery processes. The same treatment was carried out with control cultures. All experimental cultures were diluted by complete medium to obtain the same cell concentration as that at the beginning of the experiment.

\subsection{Starch quantification}

The procedures for cell disintegration, extraction of pigments and starch hydrolysis followed by spectrophotometric measurements were the same as described by Brányiková et al. (2011).

\subsection{Lipid visualization and quantification}

\subsubsection{Visualization of lipids using Nile Red fluorescence}


Intracellular lipid droplets were stained using the neutral lipid specific dye, Nile Red (9diethylamino-5H-benzo(a)phenoxazine-5-one), following the protocol described earlier (Eltgroth et al., 2005) with slight modifications. Briefly, $1 \mathrm{~mL}$ of the cell suspension was fixed with glutaraldehyde at a final concentration of $0.25 \%(\mathrm{v} / \mathrm{v})$ and stained with $4 \mu \mathrm{L}$ of

Nile Red (Sigma, N3013) stock solution $\left(0.5 \mathrm{mg} \mathrm{mL}^{-1}\right.$ of acetone) that was stored in the dark at $4{ }^{\circ} \mathrm{C}$. Samples were observed after 10 min using an epifluorescence OLYMPUS BX 51 (Olympus, Tokyo, Japan) microscope equipped with the filter combination U-MNU2 (360$370 \mathrm{~nm}$ excitation and $>420 \mathrm{~nm}$ emission). Photomicrographs were taken with a digital camera DP72, and processed using Adobe Photoshop 7.

\subsubsection{Nile Red fluorescence determination of lipids}

The algal suspension was fixed with glutaraldehyde to a final concentration of $0.25 \%$ (v/v) and loaded into wells (100 $\mu \mathrm{L}$ per well) of a $96-w e l l$ plate. $4 \mu \mathrm{L}$ of Nile Red solution were added to wells, and the fluorescence intensity was measured using a 96-well plate luminometer (Tecan infinite 200, Switzerland) with the following filters: excitation $485 \mathrm{~nm}$ (bandwidth $20 \mathrm{~nm}$ ), emission $595 \mathrm{~nm}$ (bandwidth $10 \mathrm{~nm}$ ). Glyceryl trioleate (Sigma, T7140) was used as a lipid standard to obtain a calibration curve.

\subsubsection{Gravimetric lipid determination}

Algal cultures were harvested by centrifugation at $4000 \mathrm{~g}$ for $5 \mathrm{~min}$. Cell pellets were stored frozen at $-20^{\circ} \mathrm{C}$. Before analysis, cell pellets were dried at $50{ }^{\circ} \mathrm{C}$ for at least 3 days. The dried biomass (approximately $0.03 \mathrm{~g}$ ) was ground to a powder and placed into the thimble of a Soxhlet extractor EZ 100 H (Labor-Technik, Düsseldorf, Germany) for reflux 
lipid extraction using $100 \mathrm{~mL}$ of chloroform at $100{ }^{\circ} \mathrm{C}$ for $4 \mathrm{~h}$. The extract was then distilled at $60{ }^{\circ} \mathrm{C}$ to recover the chloroform; the residue was dried at $40{ }^{\circ} \mathrm{C}$ for $1 \mathrm{~h}$, cooled to room temperature and then weighed. The lipid content was calculated by dividing the residue weight by the dry weight before extraction.

\subsection{Determination of pigment content}

The algal suspension $(10 \mathrm{~mL})$ was centrifuged at $4000 \mathrm{~g}$ for 3 min and the sediment was collected. Phosphate buffer, $7.7 \mathrm{pH}(1 \mathrm{~mL})$, a pinch of $\mathrm{MgCO}_{3}$, and Zircon beads $(500 \mu \mathrm{L}$, diameter $0.7 \mathrm{~mm}$ ) were added to the sediment, which was then disintegrated by vortexing (Vortex Genie 2, Scientific Industries, Inc., USA) for $10 \mathrm{~min}$. Acetone (4 mL, $100 \%$ ) was added, mixed well and centrifuged at $4000 \mathrm{~g}$ for $3 \mathrm{~min}$. The supernatant was drained into a calibrated test tube using an exhauster/air pump, closed with a stopper and left standing in a dark-block. Another $4 \mathrm{~mL}$ of acetone ( $80 \%$ ) were added to the sediment, mixed well and centrifuged at $4000 \mathrm{~g}$ for $3 \mathrm{~min}$. Using an exhauster/air pump, the supernatant was drained off to the same calibrated test tube used in the preceding step and topped up with $80 \%$ acetone to $10 \mathrm{~mL}$. Optical densities at $750,664,647,470$, and $450 \mathrm{~nm}$ were measured in a $1 \mathrm{~cm}$ path length cuvette using a Shimadzu UV-1800 spectrophotometer (Shimadzu, Kyoto, Japan). Calculation of chlorophyll content was based on optical density at different wavelengths and was carried out according to equations published previously (MacKinney, 1941).

\section{Results and discussion}

\subsection{Effect of mineral medium depletion and replenishment on chlorophyll content}


The green color of the microalgal suspension, seen at the beginning of the experiment (Fig. 1A), was yellowish after 7.5 days of growth in 0.1 medium and yellow-green 1 day later in 0.2 medium (Fig. 1B, vessels 0.1 and 0.2 ), while cultures grown in complete medium became dark-green during continuous growth (Fig. 1B, vessel 1). These color changes, from dark green to yellow-green, observed in photobioreactors in which the nutrient concentration was 5 and 10 times lower than in the control medium, are in agreement with results obtained in studies of nutrient limitation by other authors using different microalgal species (Rodolfi et al., 2009; Siaut et al., 2011).

When cells cultured in 0.2 and 0.1 media for 7.5 and 9 days respectively were transferred into complete medium (medium 1) and diluted to the same cell density (Fig. 1C), the yellowgreen $(0.2$ medium $)$ and yellowish ( 0.1 medium) color as seen in Fig. 1B, changed to green within 24 h (Fig. 1D).

Changes in chlorophyll content in experimental variants illustrated in Fig. 1 are described in Fig. 2. In the control culture, a concentration of mineral medium was chosen that enabled cell growth to a concentration of about $7 \mathrm{~g} \mathrm{~L}^{-1}$ of DW (see Fig. 3A). Microalgae cultivated in complete medium (Fig. 2A) accumulated chlorophyll very rapidly, attaining a maximum concentration of about $85 \mathrm{mg} \mathrm{L}^{-1}$ within 1.5 days. Thereafter, the chlorophyll content remained constant. After 3 days, the suspension was diluted about 4-fold (chlorophyll content $19 \mathrm{mg} \mathrm{L}^{-1}$ ) with fresh complete medium; this allowed recovery (Fig. 2A) to a chlorophyll content of about $85 \mathrm{mg} \mathrm{L}^{-1}$. These findings provided evidence that the inhibition of chlorophyll accumulation was caused by depletion of minerals from the medium.

In 5-fold diluted mineral medium (medium 0.2) (Fig. 2B) the chlorophyll content increased within the first 0.5 day to $33 \mathrm{mg} \mathrm{L}^{-1}$ and $3.4 \mathrm{pg} \mathrm{cell}^{-1}$ (about the same value as in the control culture (Fig. 2A)). Similarly, in 10-fold diluted mineral medium (medium 0.1), chlorophyll accumulation occurred only for 0.5 day, but reached a much lower content (12 
mg L $\mathrm{L}^{-1}$ or $0.6 \mathrm{pg}$ cell-1 (Fig. 2C)). In both experimental variants, chlorophyll concentrations decreased continuously over the cultivation period, to a very low value (about $7 \mathrm{mg} \mathrm{L}^{-1}$ of DW or $0.2 \mathrm{pg} \mathrm{cell}^{-1}$ ). This level was attained after about 7 days in 0.2 medium (Fig. 2B) and after 5 days in 0.1 medium (Fig. 2C).

The fact that in 0.2 medium, a higher chlorophyll concentration was achieved in the first 0.5 day is probably explained by a higher initial nutrient concentration (two fold concentrated) compared with 0.1 medium. This difference in nutrient concentration in the medium could also be the reason why cells cultivated in 0.1 medium achieved a minimum chlorophyll concentration $\left(0.2 \mathrm{pg} \mathrm{cell}^{-1}\right) 2$ days before the cells cultivated in 0.2 medium.

This decrease in pigment content, known as chlorosis, was also reported in several previous studies as a result of nutrient limitation or depletion (the authors mainly studied nitrogen limitation) and high light intensities in different microalgal species and strains (Mandalam and Palsson, 1998; Rodolfi et al., 2009). These studies suggested that under nitrogen deprivation, at suitable irradiances, photosynthesis continues, although at a reduced rate and with a reduction in chlorophyll. A low magnesium concentration could also contribute to these results since it is known that photosynthetic organisms, including microalgae, need magnesium to synthesize chlorophyll. Mandalam and Palsson (1998) reported that microalgae lost chlorophyll when grown in magnesium-deficient cultures. In $P$. kessleri, the absence or low concentration of either sulfur or phosphorus caused chlorosis, even if the nitrogen concentration was adequate. Similarly to nitrogen depletion, phosphorus or sulfur depletion resulted in the overproduction of storage lipids (Li et al., 2013). Another study, dealing with the effects of nutrient depletion on lipids in Chlorella vulgaris showed that when both nitrate and phosphate ions (sulfur was not tested) were depleted simultaneously within 2 days, lipid accumulation followed (Přibyl et al., 2012). Since, in the current study, 5- and 10-fold diluted medium was used, it is very likely that the decrease in 
chlorophyll content was due to the combined and concomitant effects of reduced concentrations of several elements in the mineral medium (nitrogen, phosphorus and sulfur but also other elements such as magnesium or iron could be considered). Ledford and Niyogi (2005) explained the decrease in pigment concentration with nutrient shortage as being due to disturbances in cellular anabolic processes that require these nutrients. Subsequently, the energy demand for anabolism will decrease, as will the need for energy supplied by photosynthesis.

The transfer of depleted microalgal cells into complete medium (medium 1) (Figs 2A and B) caused a rapid synthesis of chlorophyll from about $0.2 \mathrm{pg}^{\text {cell }}{ }^{-1}$ to levels as high as $3.6 \mathrm{pg}$ cell $^{-1}$ within 1 day in the variant with 0.2 medium and 2 days in the variant with 0.1 medium.

\subsection{Effect of depletion and replenishment of mineral medium on cell number, cell size, and} dry weight

The growth of cells in complete mineral medium, during the first 3 days, was not limited and attained $7.45 \mathrm{~g} \mathrm{~L}^{-1}$ of DW and a cell concentration of $40 \times 10^{6} \mathrm{~mL}^{-1}$ (Fig. 3A). Because the concentration of minerals in the medium was chosen to enable growth to about $7 \mathrm{~g} \mathrm{~L}^{-1}$ of DW, the culture was diluted with fresh mineral medium on the $3^{\text {rd }}$ day of the culture. This allowed the cells to continue growing, attaining $7.50 \mathrm{~g} \mathrm{~L}^{-1}$ of DW and a cell concentration of $30 \times 10^{6} \mathrm{~mL}^{-1}$ within two days (Fig. 3A).

In cultures grown in diluted media (Figs 3B, C), the increase in both cell number and dry weight, in both 0.2 medium (Fig. 3B) and 0.1 medium (Fig. 3C), started at about the same rate as in the control culture, but after a short period (1.5 and 1 day respectively), the time course in DW gain tended to slow until day 9 in 5-fold diluted culture (Fig. 3B, curves DW and No) or became stationary at about day 4 in the case of 10-fold diluted culture (Fig. 3C, 
curves DW and No). Thereafter, a low (Fig. 3B) or no (Fig. 3C) increase in cell number indicated a blockage of cellular division. It has been reported that growth of C. pyrenoidosa continued very slowly for some time in a medium that was depleted or deficient in nutrients, particularly nitrogen or sulfur, and that cell division was then blocked after a short period under these conditions (Hase, 1962). A similar effect was reported most recently in the closely related species C. vulgaris (Přibyl et al., 2012; Přibyl et al., 2013).

After depletion of diluted media (media 0.2 or 0.1 ), the transfer of $P$. kessleri cells into complete medium (medium 1) markedly affected all growth characteristics (Fig. 3B and C). An increase in dry weight occurred within 1 day, followed shortly by an increase in cell number. The sharp increase in cell size, concomitant with dry weight, but not cell number and its decrease upon cell division (Figs 3B and C, curves V, DW, No), occurred shortly after transfer into complete medium. This indicated that the cells started to grow synchronously to the size of mother cells and then divided into daughter cells. The concomitant increase in chlorophyll (Figs 2B, C) supports the evidence that the cells rapidly recovered (within 2 days) from starvation stress.

\subsection{Effect of depletion and replenishment of mineral medium on starch and lipid content}

In order to monitor the photosynthetic carbon flow into different storage compounds, starch and lipid content of P. kessleri were measured under the culture conditions previously described. To distinguish between lipid forms, a gravimetric method using Soxhlet extraction was used, extracting all cellular lipid forms, including storage lipids, from disintegrated cells (total lipids). To determine neutral (storage) lipids separately, binding to Nile Red, the fluorescence dye specific for neutral lipids, was measured. By subtracting the values for 
storage lipids from values for total lipids, the content of other cellular lipids could be determined (see differences between total lipids and storage lipids in Fig. 4).

Control P. kessleri cells grown in complete mineral medium (medium 1) accumulated basal amounts of starch ( $2-5 \%$ of DW), total cellular lipids ( $6-10 \%$ of DW) and storage lipids (2 $-5 \%$ of DW) (Fig. 4A). Lipid content did not change significantly over the 10 days of cultivation, while starch increased significantly during the first 0.5 day. Since the nutrient concentration in the medium was sufficient to prevent cell stress, from 1.5 days on, there were no major changes in the concentrations of storage materials (Fig. 4A).

Cells grown in 0.2 medium showed quite different patterns of starch and lipid accumulation compared to that observed in cells grown in medium 1. In cells cultured in medium 1 , starch began to decrease sharply, whereas cells grown in 0.2 medium maintained very high levels of starch ( $25 \%$ of DW), which only decreased slightly by day 3 , and then decreased slowly over the following 6 days to about $10 \%$ of DW. Likewise, the storage lipid content remained at a constant very low level until day 3. From then onwards, the concentration of storage lipids increased continuously up to day 9 , while the starch content followed the opposite path until day 7 and then remained almost constant until day 9 (Fig. 4B).

The variation in starch and lipid content in P. kessleri cells cultivated in 0.1 medium (Fig. 4C) was similar to that seen in Fig. 4B. However, the time scale over which the variations took place was different. In this case, the decrease in starch and increase in storage lipids commenced on day 1.5 , i.e. 1.5 days earlier than in the 0.2 medium. Since the initial nutrient concentration in 0.1 medium was 2 fold lower than in 0.2 medium, nutrient limitation was reached earlier than in 0.2 medium.

The decrease in starch content and the increase in storage lipid content were much sharper than that observed in 0.2 medium. After day 1.5 , the starch content decreased steadily 
until day 6 and the lipid content increased continuously over the 7.5 days of culture. The final content of starch and lipid were quite similar to those seen in medium 0.2 . Although by using 0.1 medium it was possible to obtain the same storage lipid content (up to $30 \%$ of DW) in fewer days, the biomass obtained by cultivation in 0.2 medium (about $6 \mathrm{~g} \mathrm{~L}^{-1}$, Fig. 3B) was higher than that obtained in 0.1 medium $\left(3.5 \mathrm{~g} \mathrm{~L}^{-1}\right.$, Fig. 3C), and resulted in a higher lipid productivity $\left(0.20\right.$ versus $0.14 \mathrm{~g}$ of storage lipid $\left.\mathrm{L}^{-1} \mathrm{~d}^{-1}\right)$.

Similarly to 0.2 medium (Fig. 4B), the starch content in 0.1 medium (Fig. 4C) decreased slowly over the period of greatest increase in storage lipid, and total lipids increased or remained constant. However, although total lipids were constant, the lipids accumulating in lipid bodies continued to increase and became the major lipid fraction of the cells.. After placing the cells in complete medium (Figs 4B and C), storage lipids were rapidly degraded over one day in both experimental variants. Starch degradation was delayed somewhat in 0.2 medium (Fig. 4B), but it was also very rapid.

The starch content decreased over time, concomitant with increased storage lipid content, in agreement with the results of several authors who described the photosynthetic carbon flow into these two storage compounds (Li et al., 2011; Mizuno et al., 2013). Mizuno et al. (2013) also observed that under sulfur-deficient conditions, a sequential accumulation of starch and lipid occurred in Chlorella and Parachlorella species. Li et al. (2011) observed that the microalga Pseudochlorococcum, which used starch for the primary storage of carbon and energy, shifted carbon partitioning into neutral lipid as a secondary storage product when nitrogen was depleted for an extended period of time. Roessler (1990) explained that this shift from the primary carbon storage product (starch) to a secondary storage product (storage lipids) was due to the fact that these storage lipids are composed primarily of saturated and monounsaturated fatty acids, which can be efficiently packed into the cell and thereby generate more energy upon oxidation than starch; this represents the best storage compound 
for rebuilding the cell following stress conditions. But this is not the only strategy generally used by algae under nutrient stress. There are strains of the microalga Chlorella (Brányiková et al., 2011) and also the marine microalga Tetraselmis (Yao et al., 2013), where starch is the primary carbon storage product, even under starvation conditions (e.g. nitrogen or sulfur free medium), when the synthesis of starch rather than lipids is markedly increased.

In the present study, $P$. kessleri cells shifted from starch to lipid accumulation after 3 days of cultivation (Fig. 4B), suggesting that the nutrient concentrations (particularly nitrogen) on the third day were already at levels that induced a change in carbon partitioning. This is supported by the findings that the starch content began to decrease at that time.

The decreasing content of starch during depletion of the mineral medium shown in Figs 4B and $\mathrm{C}$ could indicate that the starch was being converted into storage lipids. Previous results supported this idea in the microalga Chlamydomonas reinhardtii (Siaut et al., 2011). It is necessary, however, to take into account the fact that concomitant with a decrease in starch content, growth and cell division (0.2 medium), and cellular maintenance, (medium 0.1) processes that exclusively use starch as an energy and carbon source occur during the time of nutritional depletion. Moreover, a large increase in storage lipid content could be detected between days 7 and 9 , i.e. during the period when starch content remained practically constant, suggesting that storage lipids were not being produced at the expense of starch. Alternative pathways that convert membrane lipids to TAGs have been demonstrated in plants and yeast (Arabolaza et al., 2008). Later this was also confirmed in the microalga, Chlamydomonas reinhardtii (Siaut et al., 2011). Additionally, P. kessleri cells cultivated in 0.2 medium produced greatly increased levels of storage lipids for two days at the end of growth in depleted medium; storage lipids thus became the predominant lipid fraction, i.e. the total lipid content stabilized and the storage lipid content continued to increase until it became a major fraction of all lipids present in these cells (Fig. 4B). The same happened in 
cells grown in 0.1 medium but earlier, at day 4 (Fig. 4C). Rodolfi et al. (2009) suggested that cellular lipid accumulation during stress conditions might derive from newly fixed carbon. The capacity for de novo lipid synthesis seems a characteristic of some oleaginous microalgae, which, when grown under stress conditions, channel the excess of carbon and energy into storage lipid (mainly TAGs) (Breuer et al., 2012; Li et al., 2011).

The results presented in Fig. 4B suggest that storage lipid accumulation can be mostly due to de novo lipid synthesis but during prolonged nutrient deficiency, the conversion of other cellular lipids into storage forms can also occur.

After placing the cells in complete medium (medium 1), a sharp decline in the levels of lipid and starch was observed (Figs 4B and C). The starch and lipid content tended towards the final values observed in Fig. 4A, where the cells were grown in complete medium during the whole experiment. This large decrease in storage compounds coincided with the observed large increase in dry weight, cell number and size (Figs 3B and C) and an increase in chlorophyll content (Figs 2A and B).

Cells recovered growth shortly after replenishing the medium and grew more or less synchronously into large mother cells cells (Figs 3B and C). Thereafter, they divided synchronously and the large lipid bodies decreased in size, becoming fragmented into numerous small ones. The results indicate that after cultivation in depleted medium, $P$. kessleri cells are able to recover their characteristics rapidly if they are placed in a complete mineral medium.

Similar recovery of growth and a decrease in lipid content and fragmentation of lipid bodies was described in C. vulgaris after transfer into complete medium. Moreover, these events can occur even under dark conditions (Přibyl et al., 2013). This finding indicates that high levels of storage compounds that accumulated during starvation can serve as an exclusive source of energy and carbon for recovery of growth and reproductive processes. 


\section{Conclusions}

Parachlorella kessleri synthesized starch as a primary storage form of carbon and energy. Starch content decreased more slowly under nutrient limiting conditions than in control cultures because energy-requiring growth and reproductive processes were slowed. The hypothesis that starch is converted into storage lipids was not supported and storage lipids were shown to be synthesized mostly de novo. Cells recovered growth and division in replenished medium, utilizing both lipids and starch as sources of energy and carbon. The microalga Parachlorella kessleri therefore has the capacity for lipid overproduction induced by medium dilution.

\section{Acknowledgements}

This study was supported by grant CREST of Japan Science and Technology Agency, by grant No. LH12145 of Ministry of Education, Youth and Sports of the Czech Republic, by grant for International Collaboration Academy of Sciences of the Czech Republic No. M200201205 and by the Technology Agency of the Czech Republic, project no: TE01020080.

\section{References}

1. Arabolaza, A., Rodriguez, E., Altabe, S., Alvarez, H., Gramajo, H., 2008. Multiple pathways for triacylglycerol biosynthesis in Streptomyces coelicolor. Appl. Environ. Microbiol. 74, 2573-2582.

2. Behrens, P.W., Kyle, D.J., 1996. Microalgae as a source of fatty acids. J. Food Lipids 3, 259-272. 
3. Brányiková, I., Maršálková, B., Doucha, J., Brányik, T., Bišová, K., Zachleder, V., Vítová, M., 2011. Microalgae-novel highly efficient starch producers. Biotechnol. Bioeng. 108, 766-776.

4. Breuer, C., K., M., Kawamura, A., Takahashi, N., Ishida, T., Umeda, M., Grotewold, E., Sugimoto, K., 2012. Transcriptional repression of the APC/C activator CCS52A1 promotes active termination of cell growth. EMBO J. 31, 4488-4501.

5. Douskova, I., Doucha, J., Livansky, K., Machat, J., Novak, P., Umysova, D., Zachleder, V., Vitova, M., 2009. Simultaneous flue gas bioremediation and reduction of microalgal biomass production costs. Appl. Microbiol. Biotechnol. 82, 179-185.

6. Dragone, G., Fernandes, B.D., Abreu, A.P., Vicente, A.A., Teixeira, J.A., 2011. Nutrient limitation as a strategy for increasing starch accumulation in microalgae. Appl. Energy $88,3331-3335$

7. Eltgroth, M.L., Watwood, R.L., Wolfe, G.V., 2005. Production and cellular localization of neutral long-chain lipids in the haptophyte algae Isochorysis galbana and Emiliania huxleyi. J. Phycol. 41, 1000-1009.

8. Fernandes, B., Dragone, G., Abreu, A.P., Geada, P., Teixeira, J., Vicente, A., 2012. Starch determination in Chlorella vulgaris - a comparison between acid and enzymatic methods. J. Appl. Phycol. 24, 1203-1208.

9. Hase E., 1962. Cell division. In: R. A. Lewin (ed.), Physiology and biochemistry of algae. Academic Press, New York, pp. 617-624.

10. Heredia-Arroyo, T., Wei, W., Hu, B., 2010. Oil accumulation via heterotrophic/mixotrophic Chlorella protothecoides. Appl. Biochem. Biotechnol. 162, 1978-1995.

11. Hsieh, C.-H., Wu, W.-T., 2009. Cultivation of microalgae for oil production with a cultivation strategy of urea limitation. Bioresour. Technol. 100, 3921-3926. 
12. Hu, Q., Sommerfeld, M., Jarvis, E., Ghirardi, M., Posewitz, M., Seibert, M., Darzins, A., 2008. Microalgal triacylglycerols as feedstocks for biofuel production: perspectives and advances. Plant J. 54, 621-639.

13. Chen, C.-J., Yeh, K.-L., Aisyah, R., Lee, D.-L., Chang, J.-C., 2011. Cultivation, photobioreactor design and harvesting of microalgae for biodiesel production: A critical review. Bioresour. Technol. 102, 71-81.

14. Izumo, A., Fujiwara, S., Oyama, Y., Satoh, A., Fujita, N., Nakamura, Y., Tsuzuki, M., 2007. Physicochemical properties of starch in Chlorella change depending on the $\mathrm{CO}_{2}$ concentration during growth: Comparison of structure and properties of pyrenoid and stroma starch. Plant Sci. 172, 1138-1147.

15. Krienitz, L., Hegewald, E.H., Hepperle, D., Huss, V.A.R., Rohr, T., Wolf, M., 2004. Phylogenetic relationship of Chlorella and Parachlorella gen nov (Chlorophyta, Trebouxiophyceae). Phycologia 43, 529-542.

16. Ledford, H.K., Niyogi, K.K., 2005. Singlet oxygen and photo-oxidative stress management in plants and algae. Plant Cell Environ. 28, 1037-1045.

17. Lee, D.H., 2011. Algal biodiesel economy and competition among bio-fuels. Bioresour. Technol. 102, 43-49.

18. Li, X., Přibyl, P., Bišová, K., Kawano, S., Cepák, V., Zachleder, V., Čížková, M., Brányiková, I., Vítová, M., 2013. The microalga Parachlorella kessleri - a novel highlyefficient lipid producer. Biotechnol. Bioeng. 110, 97-107.

19. Li, Y., Han, D., Sommerfeld, M., Hu, Q., 2011. Photosynthetic carbon partitioning and lipid production in the oleaginous microalga Pseudochlorococcum sp. (Chlorophyceae) under nitrogen-limited conditions. Bioresour. Technol. 102, 123-129. 
20. Li, Y.T., Han, D.X., Hu, G.R., Sommerfeld, M., Hu, Q.A., 2010. Inhibition of starch synthesis results in overproduction of lipids in Chlamydomonas reinhardtii. Biotechnol. Bioeng. 107, 258-268.

21. Liu, Z.Y., Wang, G.C., Zhou, B.C., 2008. Effect of iron on growth and lipid accumulation in Chlorella vulgaris. Bioresour. Technol. 99, 4717-4722.

22. MacKinney, G., 1941. Absorption of light by chlorophyll solutions. J. Biol. Chem. 140, 315-322.

23. Mandalam, R.K., Palsson, B., 1998. Elemental balancing of biomass and medium composition enhances growth capacity in high-density Chlorella vulgaris cultures Biotechnol. Bioeng. 59, 605-611.

24. Mizuno, Y., Sato, A., Watanabe, K., Hirata, A., Takeshita, T., Ota, S., Sato, N., Zachleder, V., Tsuzuki, M., Kawano, S., 2013. Sequential accumulation of starch and lipid induced by sulfur deficiency in Chlorella and Parachlorella species. Bioresour. Technol. 129, $150-155$.

25. Přibyl, P., Cepák, V., Zachleder, V., 2012. Production of lipids in 10 strains of Chlorella and Parachlorella, and enhanced lipid productivity in Chlorella vulgaris. Appl. Microbiol. Biotechnol. 94, 549-561.

26. Přibyl, P., Cepák, V., Zachleder, V., 2013. Production of lipids and formation and mobilization of lipid bodies in Chlorella vulgaris. J. Appl. Phycol. 25, 545-553.

27. Rawsthorne, S., 2002. Carbon flux and fatty acid synthesis in plants. Prog. Lipid Res. 41, 182-196.

28. Rodolfi, L., Zittelli, G.C., Bassi, N., Padovani, G., Biondi, N., Bonini, G., Tredici, M.R., 2009. Microalgae for oil: strain selection, induction of lipid synthesis and outdoor mass cultivation in a low-cost photobioreactor. Biotechnol. Bioeng. 102, 100-112. 
29. Roessler, P.G., 1990. Environmental control of glycerolipid metabolism in microalgae: Commercial implications and future research directions. J. Phycol. 26, 393-399.

30. Shen, Y., Yuan, W., Pei, Z., Mao, E., 2010. Heterotrophic culture of Chlorella protothecoides in various nitrogen sources for lipid production. Appl. Biochem. Biotechnol. 160, 1674-1684.

31. Siaut, M., Cuine, S., Cagnon, C., Fessler, B., Nguyen, M., Carrier, P., Beyly, A., Beisson, F., Triantaphylides, C., Li-Beisson, Y., Peltier, G., 2011. Oil accumulation in the model green alga Chlamydomonas reinhardtii: characterization, variability between common laboratory strains and relationship with starch reserves. BMC Biotechnol. 11:7, 1-15.

32. Sunda, W.G., Price, N.M., Morel, F.M.M., 2005. Trace metal ion buffers and their use in culture studies. in: R.A. Andersen (Ed.) Algal culturing techniques. Elsevier, Amsterdam, pp. $35-63$.

33. Takagi, M., Karseno, Yoshida, T., 2006. Effect of salt concentration on intracellular accumulation of lipids and triacylglyceride in marine microalgae Dunaliella cells. $J$. Biosci. Bioeng. 101, 223-226.

34. Yang, J., Xu, M., Zhang, X., Hu, Q., Sommerfeld, M., Chen, Y., 2011. Life-cycle analysis on biodiesel production from microalgae: Water footprint and nutrients balance. Bioresour. Technol. 102, 159-165.

35. Yao, C., Ai, J., Cao, X., Zhang, W., 2012. Enhancing starch production of a marine green microalga Tetraselmis subcordiformis through nutrient limitation. Bioresour. Technol. 118, 438-444. 


\section{Figure captions}

Fig. 1 Laboratory photobioreactor used for the experiments. A: Cultures at the beginning of experiment. B: Cultures after 7.5 days of the growth. C: Cultures were transferred into complete mineral medium and diluted to have the same initial biomass concentrations. D: Recovery from starvation in complete mineral medium. Vessels in A and $\mathbf{B}$ - 1: complete mineral medium; 0.2: 5-fold diluted medium; 0.1: 10-fold diluted medium. Vessels in $\mathbf{C}$ and D - 1: complete mineral medium with cells from previous complete mineral medium; $\mathbf{0 . 2}$ : complete mineral medium with cells cultivated previously in 5-fold diluted medium; $\mathbf{0 . 1}$ : complete mineral medium with cells cultivated previously in 10-fold diluted medium.

Fig. 2 Changes in chlorophyll content in cultures of Parachlorella kessleri cultivated in complete mineral medium (medium 1) (A) or 5-fold diluted (medium 0.2) (B) or 10-fold diluted mineral medium (medium 0.1) (C). Cultures grown in diluted mineral media $(\mathbf{B}, \mathbf{C})$ were transferred into complete mineral medium (medium 1) after 9 or 7.5 days, respectively.

The chlorophyll content is expressed in $\mathrm{mg} \mathrm{L}^{-1}$ (full circles, solid line) and in pg cell ${ }^{-1}$ (empty circles, dashed line). The course of chlorophyll synthesis in control cultures (A) is also illustrated in nutrient limited cultures (B, C) (dotted line without symbols).

Fig. 3 Changes in dry weight (DW), mean cell volume (V) and cell number (No) in cultures of Parachlorella kessleri. Cultures were grown either in complete mineral medium (medium 1) (A) 5-fold diluted medium (medium 0.2) (B) or 10-fold diluted medium (medium 0.1) (C). After 3 days (A), 9 days (B), or 7.5 days (C), cells were transferred into complete mineral medium (medium 1). 
Fig. 4 Changes in relative starch, total cellular lipid and storage (neutral) lipid content in cultures of Parachlorella kessleri. Cultures were grown either in complete mineral medium (medium 1) (A), 5-fold diluted (medium 0.2) (B), or 10-fold diluted (0.1) (C) medium. After 9 days (B) or 7.5 days $(\mathbf{C})$, cells were transferred into complete mineral medium (medium 1). 

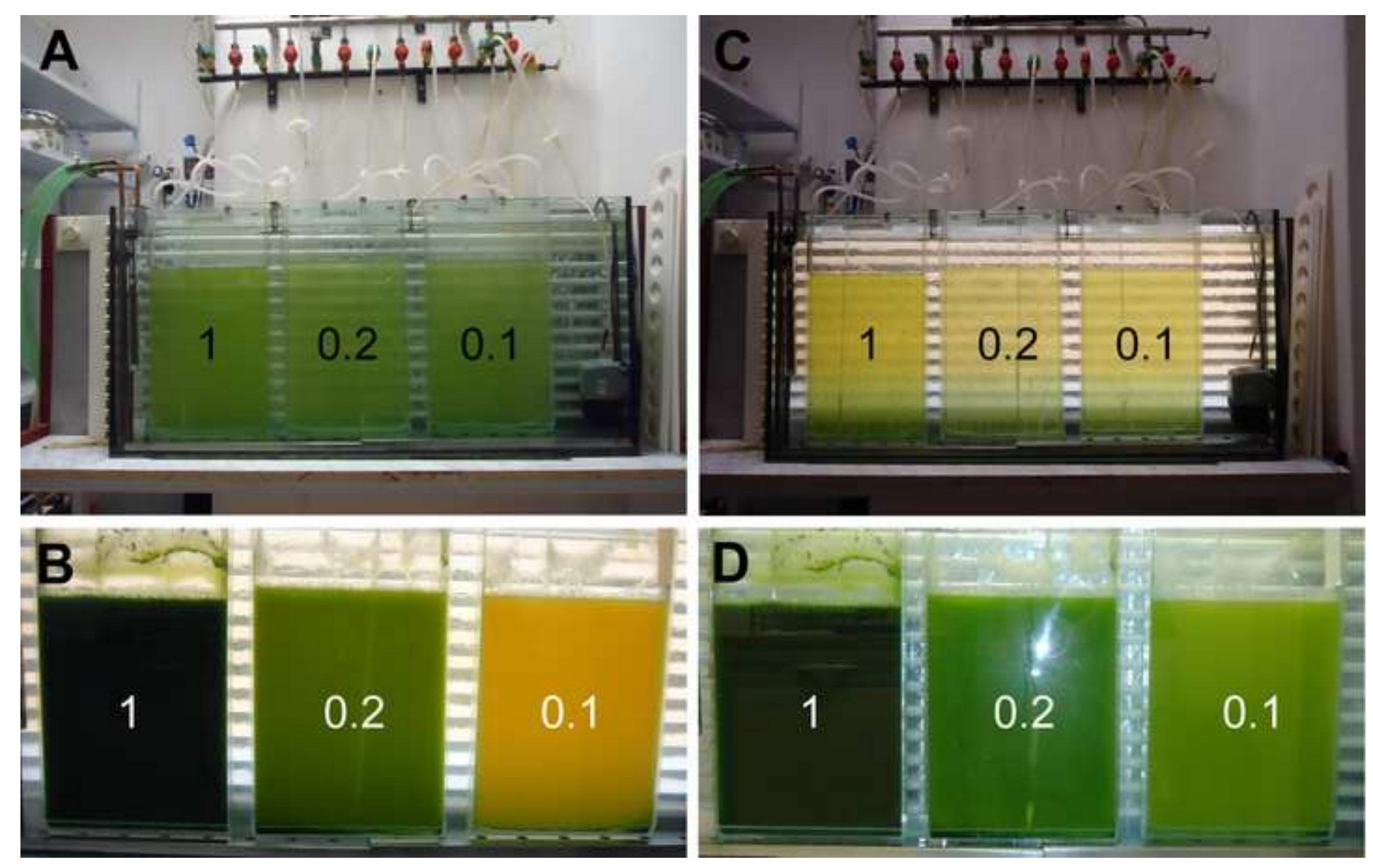


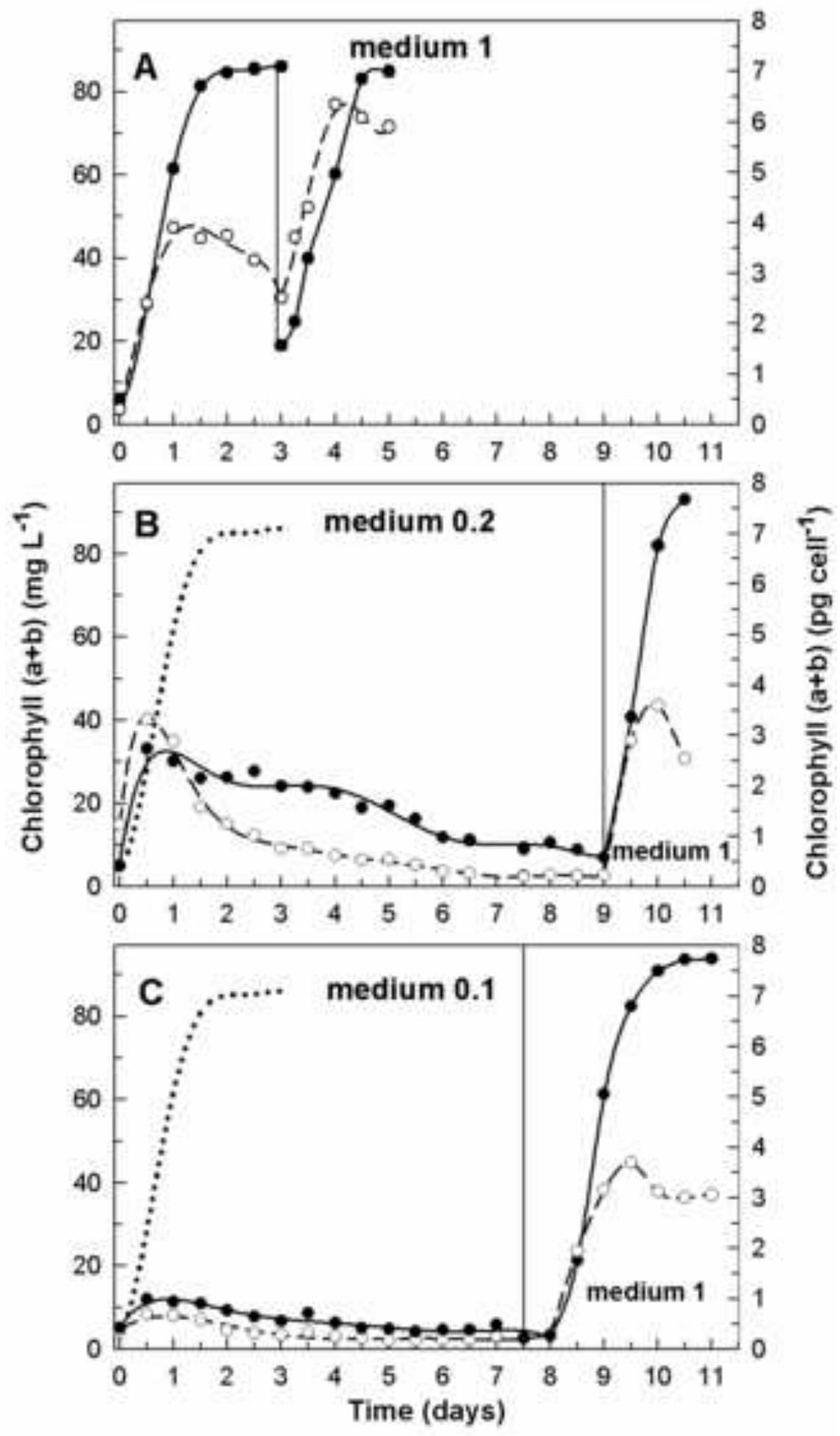




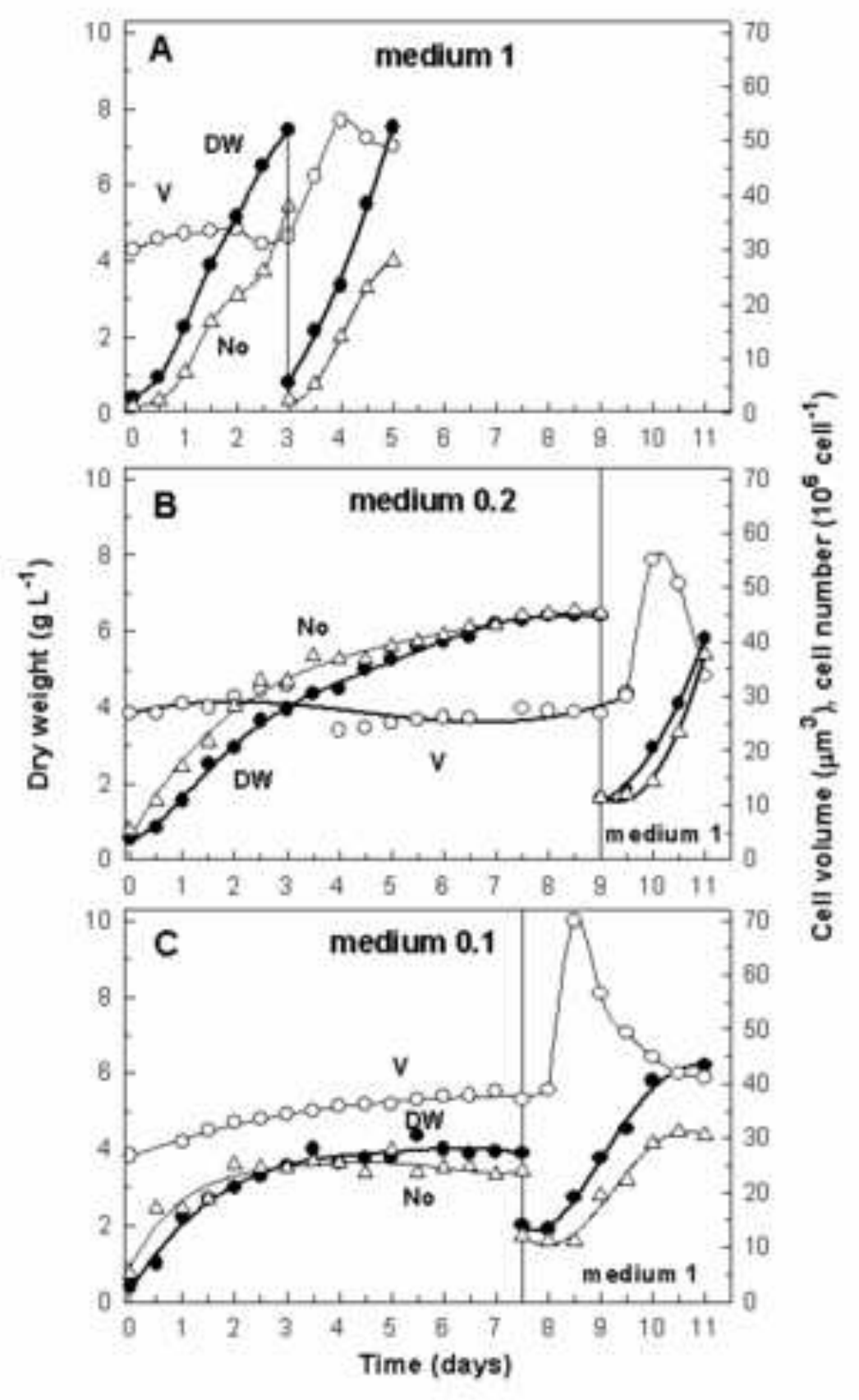

Figure 3

है

$$
\text { है }
$$




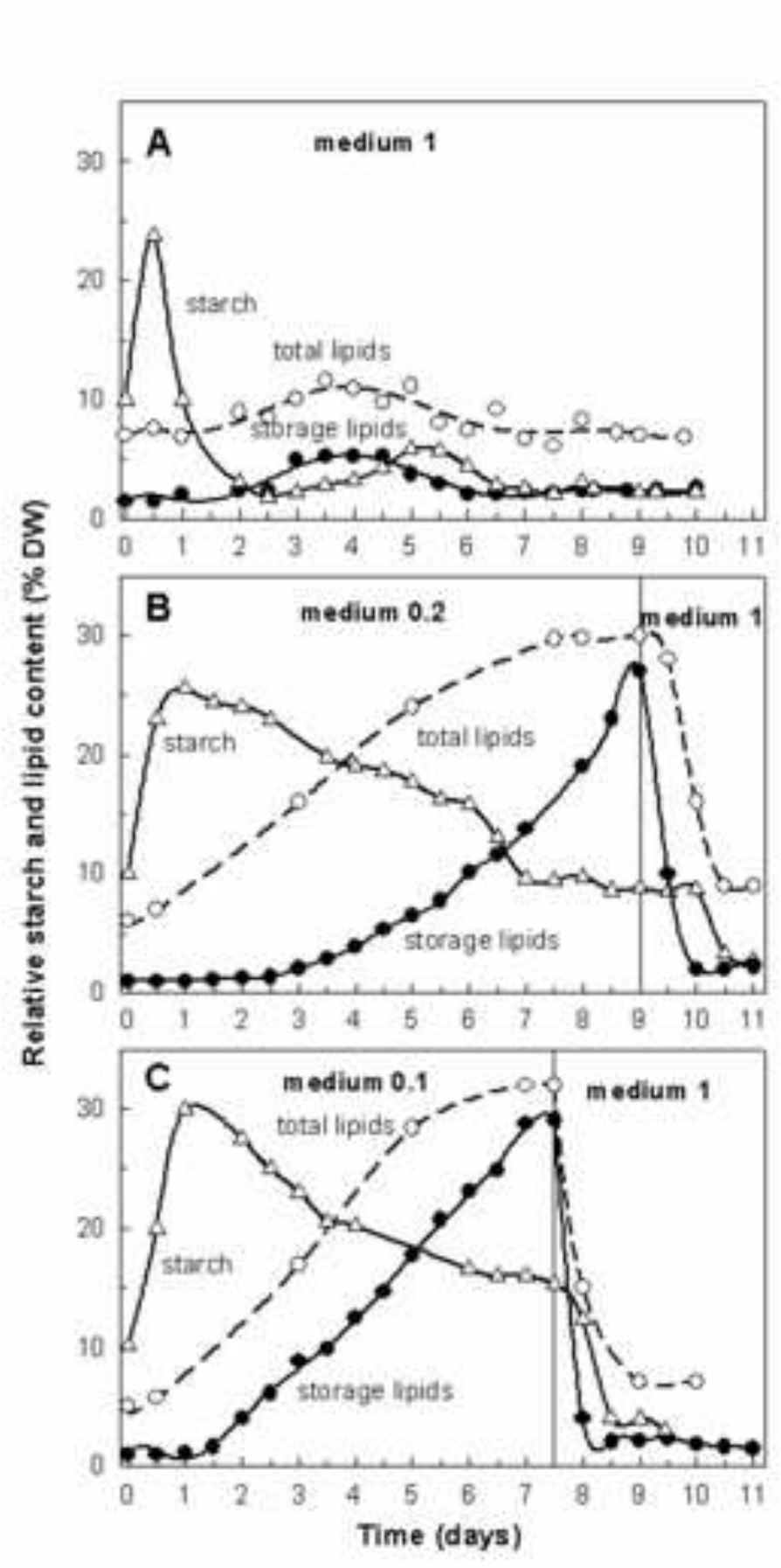

Figure 4 